University of Massachusetts Amherst

ScholarWorks@UMass Amherst

Chemistry Department Faculty Publication Series

Chemistry

1989

\title{
Study of the Possible Benefits of Flow Injection Sample Introduction for Flame Atomic Absorption Spectrometry
}

Julian Tyson

University of Massachusetts Amherst

C. E. Adeeyinwo

Loughborough University of Technology

S. R. Bysouth

Loughborough University of Technology

Follow this and additional works at: https://scholarworks.umass.edu/chem_faculty_pubs

Part of the Chemistry Commons

\section{Recommended Citation}

Tyson, Julian; Adeeyinwo, C. E.; and Bysouth, S. R., "Study of the Possible Benefits of Flow Injection Sample Introduction for Flame Atomic Absorption Spectrometry" (1989). Journal of Analytical Atomic Spectrometry. 1301.

Retrieved from https://scholarworks.umass.edu/chem_faculty_pubs/1301 


\title{
Study of the Possible Benefits of Flow Injection Sample Introduction for Flame Atomic Absorption Spectrometry*
}

\author{
Julian F. Tyson, Christina E. Adeeyinwo and Stephen R. Bysouth \\ Department of Chemistry, University of Technology, Loughborough, Leicestershire LE11 3TU, UK
}

\begin{abstract}
Flow injection techniques for use with atomic spectrometry are briefly reviewed, from which it is apparent that there has been a considerable increase in interest recently in on-line pre-concentration and matrix isolation methods. In addition to the use of immobilised reagents and solvent extraction, methods based on precipitation and collection of the precipitate in the manifold have been reported. The possible benefits of running the nebuliser under starvation conditions both with and without air compensation are examined together with the benefits of peak-area as opposed to peak-height measurements, claimed in some previous studies to lead to improved detection limits. It is shown that with a Baird Atomic A3400 nebuliser the depressive effect of phosphate on calcium may be removed at low flow-rates and that with a Philips SP9 nebuliser the best detection limits are obtained when strategies for maximising the peak height are adopted. Preliminary results for the generation of a volatile metal chelate (copper trifluoroacetylacetonate) as the basis for matrix isolation show a considerable improvement in peak-area sensitivity over conventional nebulisation, although the peak-height sensitivity is similar.
\end{abstract}

Keywords: Flow injection; flame atomic absorption spectrometry; on-line pre-concentration; matrix removal; detection limits

Although the first papers to describe flow injection (FI) sample introduction for atomic spectrometry and to use these words in their titles appeared in 1979,1.2 little interest was shown in the possibilities of the combination. In a review of the literature up to late $1984^{3}$ published after the second BNASS conference, only about 30 papers on FI atomic absorption spectrometry (FI-AAS) and ten papers on FI inductively coupled plasma atomic emission spectrometry (FI-ICP-AES) were cited.

However, by late 1985 the combination of FI with atomic spectrometry was identified as the fastest growing area of FI applications in spectroscopy ${ }^{4}$ and, in the most recent comprehensive coverage of the FI literature (up to the end of 1986), 5 atomic spectrometric applications accounted for almost $12 \%$ of the 1400 references cited. This included 123 papers on FI-AAS and 37 on FI-ICP-AES. The latter area has been reviewed separately6.7 and the developments in the entire field of FI atomic spectrometry in the last three years have been summarised. ${ }^{8}$

Flow injection techniques extend the performance of the atomic spectrometer in a number of dimensions. Samples with high dissolved solid contents, of variable viscosity and with high organic solvent contents can be introduced more successfully. The upper limit of the conventional working range may be improved by a variety of on-line dilution procedures, most of which can be adapted to the provision of calibration data (including several variants of the FI standard additions method). Interference effects may be reduced by the mode of operation of the spectrometer with respect to the sample uptake rate ${ }^{10}$ or by a variety of on-line matrix removal procedures. These procedures often act also as pre-concentration steps and thus the lower limit of the conventional working range may be extended. Solvent extraction, ${ }^{11}$ precipitation ${ }^{12}$ and solid or immobilised reagents ${ }^{13}$ have all been used.

Developments in the last area have been considerable and a wide variety of materials, some of which are commercially available, have been packed into small columns and inserted into flow lines. Several designs of manifold have been used. including a fully automated procedure, ${ }^{14}$ and it is suggested that the best design is one which allows the matrix components to flow to waste during the collection step. This can be

* Presented at the Fourth Biennial National Atomic Spectroscopy Symposium (BNASS), York, UK, 29th June-1st July, 1988. conveniently achieved if the separator column is located within the loop of an injection valve. ${ }^{15}$ Detection limits comparable to those obtainable with electrothermal atomisation AAS have been reported with this type of manifold using flame AAS (FAAS).

It has been shown that it is possible to pre-concentrate lead by precipitation with ammonia solution and collection on a stainless-steel filter followed by dissolution in nitric acid. ${ }^{12}$ If sample throughput was sacrificed, concentrations in a $250-\mathrm{ml}$ sample volume of down to $1.2 \mathrm{ng} \mathrm{ml}^{-1}$ could be determined.

All three of these on-line pre-concentration approaches have been used as the basis of indirect methods. For example, cyanide has been determined 16 by passing the carrier solution through a mini-column of copper(II) sulphide with subsequent detection of the copper released as $\mathrm{Cu}(\mathrm{CN})_{4}{ }^{2-}$, nitrate has been determined 17 by extraction of the ion pair formed with bis(2,9-dimethyl-1,10-phenanthrolinyl)copper(I) into 4methylpentan-2-one and chloride by the precipitation of silver chloride and detection of the decrease in absorbance of a silver reagent stream. 18

Vapour generation of mercury and covalent hydrides has been reported for the analysis of some real samples, including soils and plants ${ }^{19}$ and glycerine. ${ }^{20}$ This approach has considerable merits in terms of achieving the objectives of removal of matrix components from the vapour phase and increasing the atom partial pressure over conventional solution nebulisation, but suffers from potential interferences in the vapour generation reaction. As complex chemical reactions can be performed on-line using the FI approach, it is thought that this approach to sample presentation could be extended to other volatile determinant species and this is considered further in the Experimental section of this paper.

It is possible to adapt FI manifolds for speciation studies and again mini-columns of appropriate materials have been used. For example, acidic alumina has been used to separate $\mathrm{Cr}^{\text {III }}$ and $\mathrm{Cr}^{\mathrm{VI}}$ based on the selective retention of $\mathrm{Cr}^{\mathrm{VI}}$ under the operating conditions initially used, followed by stripping with a suitable eluent. ${ }^{21}$ Most of these methods bear some resemblance to gradient elution chromatography and most FI speciation studies have been described as coupled liquid chromatography - atomic spectrometry. This topic has recently been reviewed comprehensively. ${ }^{22}$

Although it may be controversial to view chromatography as a sub-set of FI methods, there is definite controversy in the 
FI-AAS literature over the best operating conditions, particularly with respect to the effect of flow-rate on sensitivity and detection limit.

Under a given set of operating conditions, e.g., oxidant flow-rate, position of the capillary tip in the venturi throat, etc., a concentric nebuliser develops a certain suction. The rate at which a solution is drawn into the nebuliser under the action of this suction depends on the dimensions of the capillary tubing, the viscosity of the solution and the hydrostatic head over which liquid has to be raised. There is therefore no such thing as "the natural flow-rate of the nebuliser." Further, it has been shown ${ }^{23}$ that the nebuliser suction depends on whether there is a thread of liquid in the capillary and on its flow-rate.

If for a given set of operating conditions and solution characteristics, etc., the uptake rate is reduced by controlling the flow with an external pump, the steady-state signal will fall as the partial pressure of atoms in the light beam decreases. This decrease, however, is not directly proportional to the reduction in the rate of analyte mass introduction into the nebuliser as the nebuliser exhibits increased efficiency under starvation conditions. In turn this means that an FI peak obtained under these starvation conditions, although having a height decreased in comparison with the steady-state signal level, exhibits an increased area. It should be borne in mind, of course, that any flow-through detector giving a response proportional to concentration will show this effect. If the response is independent of flow-rate, peak area will be inversely proportional to flow-rate.

One of the earliest papers on FI-AAS ${ }^{2}$ sought to take advantage of this increased sensitivity for peak area and further showed that an increase in precision for peak-area measurements over peak-height measurements could be obtained, leading to the calculation of lower detection limits (on a mass basis rather than a concentration basis) for the FI mode compared with the conventional mode of operation. This improved precision for peak area at lower flow-rates has been confirmed by several groups of workers. ${ }^{10,24}$ Not all workers agree that FI-AAS should be performed under starvation conditions and the use of enhanced flow-rates has been recommended. 25

The situation is further complicated by reports on the effect of deliberately introducing a compensating flow of air immediately prior to the nebuliser. Some workers have claimed that this produces an increase in precision, ${ }^{10}$ some have claimed both an increase in sensitivity and precision ${ }^{24}$ and some have found a decreased precision. ${ }^{11}$

In this paper, the possible benefits of FI sample introduction at reduced flow-rates for the reduction of stable compound interferences and for the improvement in detection limits are examined, together with the feasibility of the on-line generation of a volatile metal derivative.

\section{Experimental}

\section{Apparatus}

Three atomic absorption spectrometers were used. For the study of stable compound interferences, a Baird Atomic A3400 instrument was used, and Philips SP9 instruments were used for the study of the effect of flow-rate on detection limit and the measurement of the volatile metal chelate. For the former study the spectrometer was interfaced to an SP9 computer and for the latter the signal was monitored with a Philips PM8251 chart recorder.

Flow injection manifolds for the first two studies were as described previously 8,26 ; for the vapour generation work the manifold is shown schematically in Fig. 1. The phase separator used was of the type described by Yamomoto et al. ${ }^{27}$ and consisted of $55 \mathrm{~mm}$ of microporous PTFE tubing (Japan Gore-Tex, Tokyo, Japan) and the vaporisation was achieved by a gas chromatographic oven (Perkin-Elmer F11) at 220-
$240{ }^{\circ} \mathrm{C}$. The resulting vapour was transported by a stream of pre-heated air through a heated $\left(150^{\circ} \mathrm{C}\right)$ glass transfer line $(50$ crn long $\times 2 \mathrm{~mm}$ i.d.) which entered the end of a Perkin-Elmer burner (Model 272 instrument) approximately $20 \mathrm{~mm}$ below its top and protruded about $20 \mathrm{~mm}$ inside the burner. The air flow-rate was $11 \mathrm{~min}^{-1}$. The burner was mounted on the SP9 spray chamber and secured by a suitable diameter rubber sleeve.

For all studies the spectrometers were optimised with respect to sensitivity while introducing solutions in the conventional manner.

\section{Reagents}

Metal ion solutions were prepared by dilution of stock 1000 $\mathrm{mg} \mathrm{l}^{-1}$ solutions $(\mathrm{BDH})$. Phosphate solutions were prepared by appropriate dilution of Aristar-grade orthophosphoric acid. A $0.5 \mathrm{~m}$ sodium acetate solution was used to adjust the $\mathrm{pH}$ in the vapour generation manifold prior to merging with a reagent stream consisting of $5 \% V / V$ trifluoroacetylacetone in chloroform - ethanol $(2+1)$. Aqueous solutions were prepared in analytical-reagent grade water from a Liquipure $\mathrm{RG}$ system (reverse osmosis followed by ion exchange).

\section{Procedures}

The response of the spectrometer to a solution containing 10 mg $1^{-1}$ of calcium and a solution containing $10 \mathrm{mg}^{-1}$ of calcium with $200 \mathrm{mg} \mathrm{l}^{-1}$ of phosphate was studied as a function of flow-rate for both normal nebulisation and FI introduction, both with and without air compensation. In all instances the flow-rate was controlled by the peristaltic pump. Air compensation was achieved by the inclusion of a T-connector in the flow line immediately in front of the nebuliser.

For the precision and detection limit studies, the relative standard deviation (RSD) based on five integrations was measured at $283 \mathrm{~nm}$ as a function of flow-rate over the range $0.72-3.4 \mathrm{ml} \mathrm{min}^{-1}$ for $50 \mu \mathrm{l}$ of a $10 \mathrm{mg} \mathrm{l}^{-1}$ lead solution, both with and without air compensation. The integration time was varied between 15 and $25 \mathrm{~s}$, depending on the flow-rate. The RSD as a function of concentration for ten lead solutions covering the range $0.05-50 \mathrm{mg} \mathrm{l}^{-1}$ on the basis of five 4 -s integrations was measured for normal nebulisation and on the basis of 25-s integrations of four replicate injections at a flow-rate of $2.2 \mathrm{ml} \mathrm{min}^{-1}$ without air compensation.

After partial optimisation of the operating parameters (mainly flow-rates) for the vapour generation manifold, the absorbance - time response of the system for the injection of solutions containing 10 and $1 \mathrm{mg} \mathrm{l}^{-1}$ of copper was obtained. The response was also obtained for the direct FI introduction of the same volume of the $10 \mathrm{mg} \mathrm{l}^{-1}$ solution into the nebuliser via a single-line manifold.

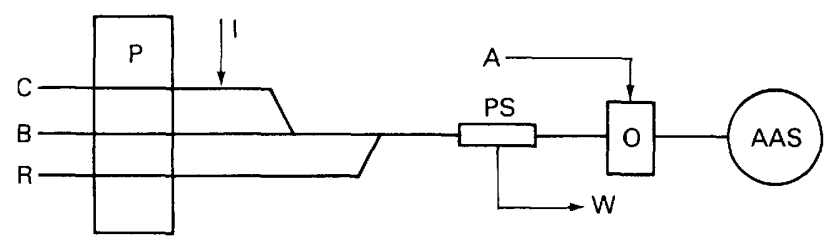

Fig. 1. Manifold for generation of copper trifluoroacetylacetonate A, Air inlet to oven; B, $0.5 \mathrm{~m}$ sodium acetate solution at $0.3 \mathrm{ml} \mathrm{min}^{-1}$; $C$, carrier stream of water at $0.14 \mathrm{ml} \mathrm{min}^{-1}$; I, injection valve, $112 \mathrm{ul}$; $O$, gas chromatography oven; $P$, peristaltic pump; PS, phase separator; $\mathrm{R}$, trifluoroacetylacetone reagent in chloroform - ethanol and W, waste. All tubing is $0.5 \mathrm{~mm}$ i.d. with lengths of $70 \mathrm{~cm}$ between the valve and the first confluence point, a further $70 \mathrm{~cm}$ to the second confluence point, $170 \mathrm{~cm}$ to the phase separator and a further $90 \mathrm{~cm}$ to the oven 


\section{Results and Discussion}

The effect of flow-rate and air compensation on the calcium signals is shown in Fig. 2. It can be seen that for both types of manifold (with and without air compensation) the extent of the depressive effect of phosphate is reduced at low flow-rates and eventually disappears. The use of air compensation appears to be beneficial as far as the reduction of the interference is concerned but causes a decrease in sensitivity. These results suggest that with this particular type of nebuliser and spray chamber the effect of reducing the flow-rate is to increase the proportion of smaller drops being transported to the flame to such an extent that the residence time of the smaller clotlets formed on evaporation of the solvent is sufficient to produce the same extent of atomisation in the presence of phosphate as in its absence. These results are in agreement with those reported by other workers. ${ }^{10,24}$

The variation of peak height and peak area with flow-rate is given in Table 1 together with the corresponding RSD values. Graphs of RSD as a function of flow-rate are given in Fig. 3 and clearly show that, as far as peak area is concerned, as the flow-rate decreases there is a considerable improvement in precision in the absence of air compensation and that at most of the flow-rates investigated the use of air compensation improves the precision. It is also clearly seen that when the precision for peak height is compared with that for peak area, a considerable decrease is observed as the flow-rate decreases so that, for most of the flow-rates studied, peak-height precision is worse than peak-area precision.

The use of air compensation appears to improve the precision for peak height, at least at the lower flow-rates, and there appears to be little to choose between peak-height and peak-area precisions when air compensation is used. As was observed with the Baird Atomic instrument, the use of air compensation reduced the peak-height sensitivity in this instance by as much as one third. Peak-area sensitivity was not affected. Again, these results are in agreement with those obtained by previous workers. 2.24 .28

However, unlike the previous reports, it is not considered possible to conclude from these results that better detection limits will be obtained when peak area is used as the quantitative basis. Although detection limits may be defined in a number of ways, in practice the detection limit is reached when the response of the instrument to a low concentration cannot be distinguished (at some appropriate level of confidence) above the background because of the noise. It is well known that the noise is related to signal strength and that for most spectrophotometric methods a plot of RSD against signal level (or concentration), sometimes referred to as a Ringbom plot, has a characteristic $U$-shape. The appropriate graphs for peak-area and peak-height measurements are shown in Fig. 4.

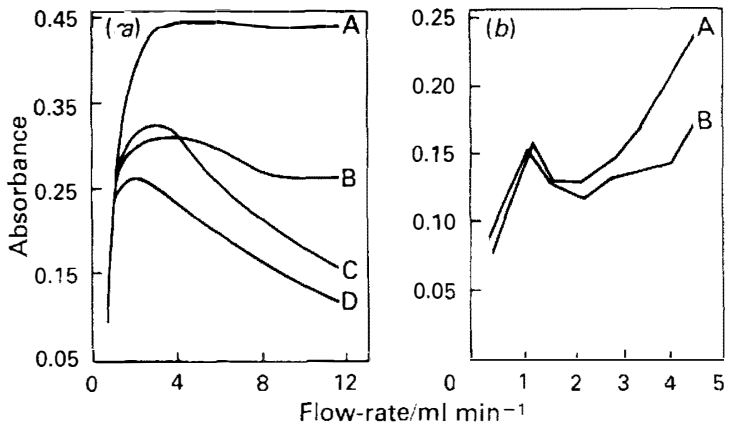

Fig. 2. Stable compound interference studies. (a) No air compensation: A, steady state for $10 \mathrm{mg}^{1^{-1}}$ calcium; B, steady state for 10 $\mathrm{mg} \mathrm{l}^{-1}$ calcium with $200 \mathrm{mg} \mathrm{l}^{-1}$ phosphate; C, FI for $10 \mathrm{mg} \mathrm{l}^{-1}$ calcium; and D, FI for $10 \mathrm{mg} \mathrm{l}^{-1}$ calcium with $200 \mathrm{mg} \mathrm{l}^{-1}$ phosphate. $(b)$ Air compensation: A, $10 \mathrm{mg} \mathrm{l}^{-1}$ calcium; and B. $10 \mathrm{mg} \mathrm{l}^{-1}$ calcium with $200 \mathrm{mg} \mathrm{l}^{-1}$ phosphate
It can be seen that although the flow-rate chosen represents a minimum in the precision versus flow-rate graph (see Fig. 3), there is little to choose between peak area and peak height with normal nebulisation. For FI introduction, the results suggest that peak-height precision may be slightly better for a portion of the concentration range. There is certainly no evidence from these graphs that the detection limits are significantly different for the two measurement modes. This suggests that for an instrument which is making a digital measurement of peak area the expected benefits of this quantitative parameter do not accrue and that when the instrument can no longer distinguish a peak height above the background noise, it can no longer distinguish a peak area.

It is possible that a major source of noise at low flow-rates is the peristaltic pump, as the pulsations become worse as the rotation speed of the pump head decreases. To some extent this can be offset by the use of simple pulse dampers, ${ }^{26}$ but carrier stream delivery from a pressurised reservoir is probably the best method of achieving a pulse-free delivery at low flow-rates. It has been reported that when such a device was used, the peak-height precision improved from being consistently worse than that for peak area to being consistently better. ${ }^{24} \mathrm{~A}$ reduction in carrier stream delivery noise may be the reason why air compensation improves the precision.

In comparing the results obtained for studies of this type with different instruments, it should be borne in mind that the performance of any given nebuliser is critically dependent on the position of the capillary tip in the venturi throat. One manufacturer, for example, states that with their design of nebuliser a movement of $0.1 \mathrm{~mm}$ in the capillary position changes the suction from zero to maximum. ${ }^{29}$ For this reason, manufacturers often set the position of the capillary as a compromise between sensitivity, detection limit and freedom from stable compound interferences, and it may therefore be possible to find operating conditions for which the sensitivity is improved over the performance of the instrument as delivered.

Recorder traces for the copper signal obtained from the vapour generation manifold are shown in Fig. 5, which also shows the conventional FI peak for injection close to the nebuliser. Although there is a considerable increase in peak area for the vapour introduction, showing a vastly improved efficiency in the over-all atomisation, the results obtained for the relative merits of peak height and peak area do not support the notion that the vapour-phase manifold will give rise to improved detection limits. However, these results are preliminary in nature and it is thought that improvements in peak-height sensitivity could be obtained by further optimisation of all the relevant parameters and a consideration of the design of the manifold - burner interface. Some of the designs already described for the gas chromatography-atomic absorption interface would be applicable to this experimental arrangement.

Table 1. Flow injection peak height and peak area as a function of flow-rate. Figures in parentheses are RSDs (\%)

\begin{tabular}{cccccc} 
& \multicolumn{2}{c}{ Peak height/mA } & & \multicolumn{2}{c}{ Peak area/mA s } \\
\cline { 2 - 3 } \cline { 5 - 5 } Flow-rate/ & No & Air & & No & Air \\
ml min $^{-1}$ & compensation compensation & compensation compensation \\
3.4 & $70(2.0)$ & $54(4.4)$ & & $106(6.2)$ & $93(18)$ \\
2.6 & $69(3.7)$ & $57(4.2)$ & & $144(4.6)$ & $131(17)$ \\
2.3 & $70(5.6)$ & $55(1.3)$ & & $146(2.3)$ & $135(11)$ \\
2.0 & $71(5.8)$ & $53(2.1)$ & & $163(4.6)$ & $168(7.6)$ \\
1.5 & $69(9.0)$ & $47(2.3)$ & & $185(4.1)$ & $170(6.0)$ \\
1.1 & $59(8.4)$ & $41(1.7)$ & $214(7.0)$ & $200(3.2)$ \\
0.72 & $48(15)$ & $32(5.0)$ & $230(15)$ & $229(8.9)$ \\
\hline
\end{tabular}




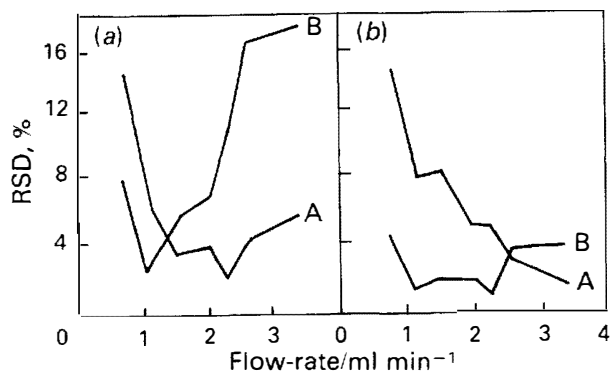

Fig. 3. Precision as a function of flow-rate. (a) Peak-area measurements; and $(b)$ peak-height measurements. A, No air compensation; and $\mathrm{B}$, with air compensation

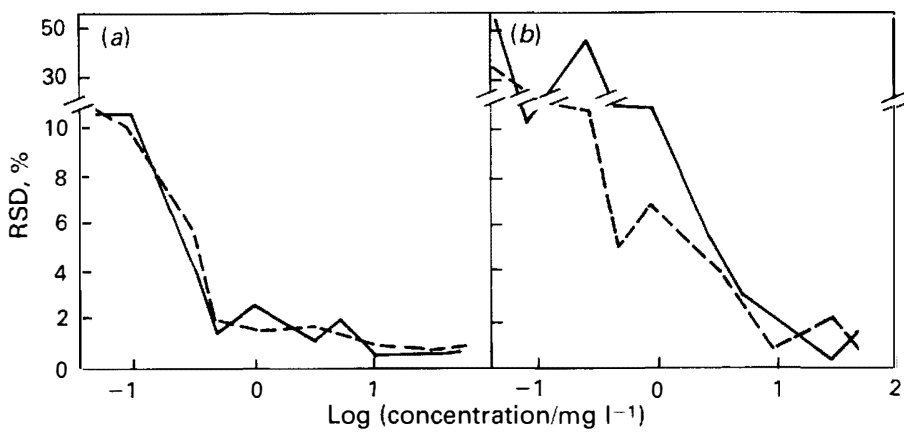

Fig. 4. Precision as a function of concentration. (a) Normal nebulisation; and $(b)$ FI introduction. Broken line, peak height; solid line, peak area

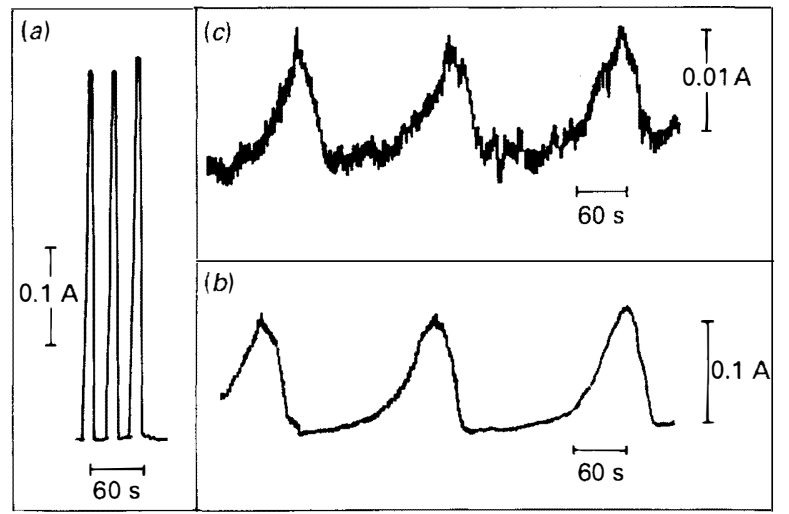

Fig. 5. Recorder traces for FI introduction of copper: (a) $10 \mathrm{mg} \mathrm{l}^{-1}$ injection $(112 \mu \mathrm{l})$ into a carrier stream close to the nebuliser; $(b) 10$ $\mathrm{mg} \mathrm{l}^{-1}$ converted into a volatile derivative; and (c) $1 \mathrm{mg} \mathrm{l}^{-1}$ converted into a volatile derivative

\section{Conclusions}

As far as optimisation of the performance of atomic absorption instruments is concerned, FI sample introduction does not produce any fundamental differences from those obtained with conventional nebulisation, in that different settings of the operating parameters are required for different figures of merit and hence it is still not possible to optimise an instrument to give simultaneously minimum interference and the best detection limit (maximum signal to noise ratio at low concentrations). The former performance requirement is aided by the use of air compensation, but detection limits are raised because of the decrease in sensitivity observed with the instruments used in this study.

When comparing the relative merits of peak-height versus peak-area measurements as a function of flow-rate, the identification of a flow-rate regime in which the RSD for peak area is lower than that for peak height does not mean the peak-area detection limits will be superior. The approach to obtaining the best detection limits for FI sample introduction is therefore to maximise the peak height and minimise the noise.

This may mean that nebulisers and spray chambers specifically designed for FI work are needed and reports of such designs are now appearing. ${ }^{30}$ One of the design features would be precise control over the position of the capillary in the venturi throat.

It is possible to generate a volatile metal chelate using an FI manifold with appropriate solvent extraction and heated tubing and to increase considerably the atomisation efficiency over conventional solution nebulisation.

Financial support for Christina Adeeyinwo from the Commonwealth Scholarship Commission and for Stephen Bysouth from the SERC is gratefully acknowledged. The authors also thank Dr. Wolfgang Frenzel of the Technichse Universität, Berlin, for helpful discussions and Dr. Akira Tanaka of Kumamoto University, who generously supplied the materials for the construction of the phase separator.

\section{References}

1. Zagatto, E. A. G., Krug, F. J.. Bergamin, Fo. H., Jorgensen, S. S. and Reis, B. F., Anal. Chim. Acta, 1979, 104, 279.

2. Wolf, W. W., and Stewart, K. K., Anal. Chem., 1979, 51, 1201.

3. Tyson, J. F., Analyst, 1985, 110, 419.

4. Růžička, J., Fresenius Z. Anal. Chem., 1986, 324, 745.

5. Růžička, J., and Hansen, E. H., "Flow Injection Analysis," Second Edition, Wiley, New York, 1988.

6. Christian, G. D., and Růžička, J., Spectrochim. Acta, Part B $1987,42,157$.

7. McLeod, C. W., J. Anal. At. Spectrom., 1987, 2. 549.

8. Tyson, J. F., Anal. Chim. Acta, 1988, 214, 57.

9. Tyson, J. F., Fresenius Z. Anal. Chem., 1988, 329, 663.

10. García, I. L., O’Grady, C., and Cresser, M. S., J. Anal. At. Spectrom., 1987, 2, 221.

11. Sweileh, J. A., and Cantwell, F. F., Anal. Chem., 1985, 57, 420 .

12. Martínez-Jiménez, P.. Gallego, M. and Valcárcel. M., Analyst, 1987, 112, 1233

13. Cox, A. G., McLeod, C. W., Miles. D. L.. and Cook, J. M., J. Anal. At. Spectrom., 1987, 2. 553.

14. Bysouth, S. R., Stockwell, P. S., and Tyson, J. F., J. Autom Chem., in the press.

15. Bysouth, S. R., Stockwell, P. S., and Tyson, J. F., Anal. Chim. Acta, 1988, 214. 329

16. Haj-Hussein, A. T., Christian, G. D., and Růžička, J., Anal. Chem., 1986, 58, 38

17. Silva, M., Gallego, M., and Valcarcel, M., Anal. Chim. Acta, 1986, 179, 341

18. MartínezJiménez, P., Gallego, M., and Valcárcel. M., J. Anal At. Spectrom., 1987, 2, 211.

19. Zhang, S., Fang, Z., and Sun, J., Guangpuxue Yu Guangpu Fenxi, 1987, 7, 57.

20. Tioh, N.-H., Israel, Y., and Barnes, R. M., Anal. Chim. Acta, 1986, 184, 205

21. Cox, A. G., Cook. I. G., and McLeod, C. W., Analyst. 1985 110,331 .

22. Ebdon, L., Hill, S., and Ward, R. W., Analyst, 1987, $112,1$.

23. O’Grady, C., Marr, I. L., and Cresser, M. S., Analyst, 1984 $\mathbf{1 0 9}, 1085$

24. Garcia, I. L., Cordoba, M. H., and Sanchez-Pedreno, C. Analyst, 1987, 112, 271.

25. Brown, M. W., and Růžička, J., Analyst, 1984, 109, 1091

26. Adeeyinwo, C. E.. and Tyson, J. F.. Anal. Chim. Acta, 1988 , 214, 339 .

27. Yamamoto, M.. Obato, Y.. Nitta, Y., Nakata, F.. and Kumamaru, T., J. Anal. At. Spectrom., 1988, 3, 441.

28. Harnly. J. M., and Beecher, G. R., J. Anal. At. Spectrom. 1986. 1. 75

29. Dymott. T. C., and Widmer. D. S.. "The Design Features of a Fully Optimised Spray Chamber for a Modern AA Instrument." Philips, Cambridge.

30. Gustavsson. A., and Nygren. -., Spectrochim. Acta, Part B. 1987, 42. 883 